\title{
High-Order Punishment and the Evolution of Cooperation
}

\author{
Bastian Baranski, Thomas Bartz-Beielstein, Rüdiger Ehlers, Thusinthan Kajendran, \\ Björn Kosslers, Jörn Mehnen, Tomasz Polaszek, Ralf Reimholz, Jens Schmidt, \\ Karlheinz Schmitt, Danny Seis, Rafael Slodzinski, \\ Simon Steeg, Nils Wiemann, Marc Zimmermann \\ Department of Algorithm Engineering \\ Dortmund University \\ D-44221 Dortmund, Germany
}

\begin{abstract}
The Prisoner's Dilemma and the Public Goods Game are models to study mechanisms leading to the evolution of cooperation. From a simplified rational and egoistic perspective there should be no altruistic cooperation in these games at all. Previous studies observed circumstances under which cooperation can emerge. This paper demonstrates that high-order punishment opportunities can maintain a higher cooperation level in an agent based simulation of the evolution of cooperation.
\end{abstract}

\section{Categories and Subject Descriptors}

H.4 [Information Systems Applications]: Miscellaneous; I.2.1 [Computing Methodologies]: Artificial IntelligenceApplications and Expert Systems[Games]; I.2.11 [Computing Methodologies]: Artificial Intelligence-Distributed Artificial Intelligence, Intelligent agents

\section{General Terms}

Experimentation

\section{Keywords}

IPD, Public Goods Game, Cooperation, Punishment

\section{INTRODUCTION}

Altruistic cooperation is common in human nature. But, from a simplified and shortsighted "rational" perspective there should be no cooperation at all. The Prisoner's Dilemma (PD) and the Public Goods Game (PGG) are often used models to study mechanisms that lead to the evolution of cooperation. In these games it is assumed that cooperation is costly to the individual and only opponents can benefit from it. The so-called free-rider problem occurs, if it is beneficial for the individual not to cooperate, no matter how the opponent reacts. Many research had been done to describe the circumstances under which cooperation can emerge despite of these drawbacks. The theory of kin-selection focus on cooperation among individuals that are in some ways closely related. Spatial structure is a factor that can sustain cooperation in a small range of PD payoff parameter [6]. The theories of direct reciprocity focus on bilateral long-term interactions [2]. The theories of indirect reciprocity explain how cooperation can emerge through reputation which directly leads to reward and punishment mechanism [7, 2].

Copyright is held by the author/owner(s).

GECCO'06, July 8-12, 2006, Seattle, Washington, USA.

ACM 1-59593-186-4/06/0007.
In the remainder of this paper we consider the tendency of individuals to punish free riders. In most cases of punishment, sending a fine has a cost to the opponent and to the punisher itself. Particularly with regard to the cost to the punisher itself, it is unclear why humans should engage in costly punishment. This leads to the so-called second-order free-rider problem.

This paper combines the agent-based model of Hammond and Axelrod [4] with punishment opportunities. We examine the effect of punishment opportunities on the evolution of cooperation and present some new results to the question "Can punishment opportunities enforce the evolution of cooperation?" Software and data from this article can be downloaded from [1].

\subsection{The Effect of Cost-Benefit Ratio}

Two agent-based models to study minimal conditions for the evolution of cooperation were proposed in [4]: (i) In the Null Model $\left(M_{\text {null }}\right)$ each agent is either an altruist who always cooperates or an egoist who never cooperates. There is no structure in the agents environment. Each time period of the model consists of four stages: immigration, interaction, reproduction, and death. The outcome of the $M_{\text {null }}$ model therefore is that no strategies based on kinship or reciprocity are produced. As expected, under these conditions there is no emergence of cooperation. (ii) The Viscosity Model $M_{\text {visc }}$ is a simple extension of the $M_{\text {null }}$ model. Viscosity means that the members of a population are spatially distributed, have limited mobility and interact locally. So the agents are now distributed on a grid. The simulation results show that viscosity sustains the evolution of cooperation in a sufficiently benign environment. It does not maintain the evolution of cooperation in an austere environment.

\subsection{First-Order Punishment in Humans}

Fehr and Gächter [3] demonstrated experimentally that punishment opportunities generate a higher average contribution level in PGG. They also observe a widespread willingness of human cooperators to punish free riders even if punishment is costly and does not provide any benefit for the punisher.

\subsection{Second- and Higher-Order Punishment in Humans}

Contributing group members who do not punish non-contributing group members are better off than those who punish, because the non-punishing group members do not pay the cost of punishing. Therefore the provision of punishment is itself a public good that is affected from the free-riding problem. The problem of cooperation ended in the problem of second-order free riders.

This may require the opportunity to punish the non-punishing group members (so called second-order punishment). However, 
this may lead to an endless regress: second-order punishment must be displaced by a third-order punishment, etc. [5].

\section{THE EXTENDED MODEL}

We extended the $M_{\text {visc }}$ and the $M_{\text {null }}$ model with high-order punishment opportunities to study possible effects on the evolution of cooperation. This model will be referred to as the extended model $\left(M_{\text {ext }}\right)$. The agents in the original agent-based model of Hammond and Axelrod used simple strategies: Each agent $A$ has a cooperation strategy, i.e., $A$ is either an altruist or an egoist. In our model we added the ability to punish egoistic and non-punishing agents: the punishment strategy. Immigration, reproduction and death stage in the $M_{\text {ext }}$ model are implemented as in the $M_{\text {visc }}$ and the $M_{\text {null }}$ model. The interaction stage in the $M_{\text {ext }}$ model is divided into the cooperation stage and the punishment stage:

Cooperation stage. Each pair of adjacent agents interacts in a onemove PD in which each agent chooses whether or not to help the other agent. Cooperative agents give help and pay a cost $C$ (denotes a decrease in the agent's potential to reproduce (PTR)) for each donation. Defective agents do not give help. Receiving help has a benefit $B$ (denotes an increase in the agent's PTR).

Punishment stage. Each agent chooses whether or not to punish egoistic or non-punishing co-players from the cooperative stage. If the simulation's global punishment level $\theta$, is set to 0 , there is no opportunity to punish other co-players. The interaction stage is finished. If $\theta=1$, agents have the opportunity to punish egoistic opponents. If $\theta=2$, agents may punish egoist opponents and directly afterwards punish non-punishing opponents. If $\theta=3$, each agent has the opportunity to punish an egoist, a non-punishing opponent, and to punish a non-punishing non-punisher opponent.

Punishing agents give a fine to the co-player and pay a cost $P$ (denotes a decrease in the agent's PTR) for each atomic punishment action. Receiving a fine has a cost $F$ (denotes a decrease in the agent's PTR).

\section{RESULTS AND ANALYSIS}

Our models use the standard parameters from Hammond and Axelrod unless otherwise stated.

Punishment Enhances the Evolution of Cooperation. The simulation results indicate that the opportunity of punishment enhances the evolution of cooperation in a more austere environment. Without punishment opportunities $(\theta=0)$ there is a total of $80 \%$ cooperative agents if $\mathrm{CBR}=0.4$. This level of cooperation can be sustained in more austere environments (i.e., $\mathrm{CBR}=0.5$ ) when the first-order punishment opportunity is given $(\theta=1)$. Regarding the higher-order punishment opportunities $(\theta=3)$, cooperation can be sustained in a much more austere environment. See Fig. 1.

Varying the Cost of Punishment. We performed simulations with varying cost-fine ratios CFR for fixed values $F=1 \%$ and $F=$ $3 \%$. The simulation results indicate that increasing the cost of punishment P-while keeping CFR constant-enforces the evolution of cooperation.

Effects in the Null Model. Hammond and Axelrod demonstrated with their $M_{\text {null }}$ model that without local interaction no cooperation can emerge in their agent-based simulation. As men-

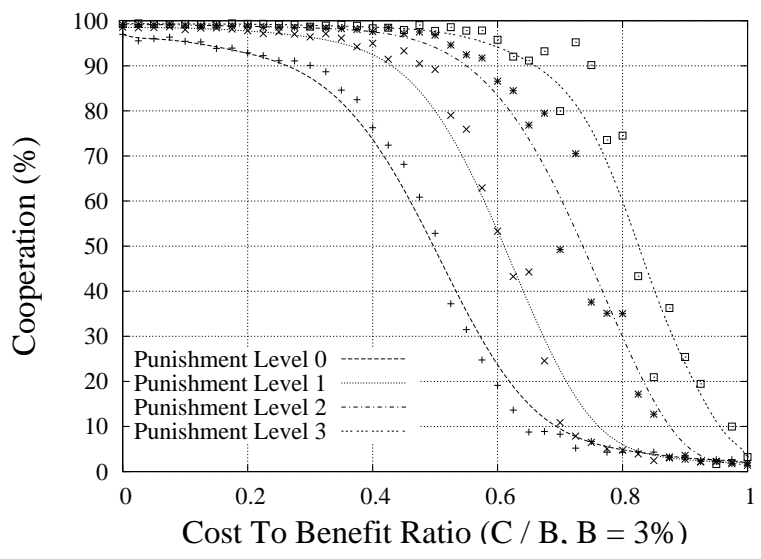

Figure 1: Higher punishment order increases the cooperation frequency. Combining the $M_{\text {vise }}$ model and the $M_{\text {ext }}$ model allows a given level of cooperation to be maintained in more austere environments. Plus, cross, star, and square symbols depict results from punishment level $\theta=0,1,2$, and 3 , respectively. Bezier curves were added to illustrate the data trend

tioned above we also extended the $M_{\text {null }}$ model with highorder punishment opportunities. The simulation results indicate that without spatial distributed agents cooperation emerges if punishing and help are not costly.

\section{SUMMARY AND OUTLOOK}

Based on careful extensions of Axelrod's and Hammond's $M_{\text {visc }}$ model, we conclude that (i) high-order punishment enables (and enforces) cooperation in austere environments, (ii) increasing the punishment $F$ values while keeping the CFR values constant enforces the evolution of cooperation, and that (iii) small CFR and CBR values support cooperation in the extended $M_{\text {null }}$ model. We have discussed various aspects of punishment strategies. Sigmund et al. [7] demonstrated how reward strategies can cause evolution of cooperation. Analyzing the interactions between reward and punishment strategies and their impact on the evolution of cooperation might be of great interest.

\section{REFERENCES}

[1] B. Baranski et al. The IPD Dortmund tournament, 2006. http://www.ipd-dortmund.de/punishment/. Cited April, 8th 2006.

[2] M. Doebeli and C. Hauert. Models of cooperation based on the prisoner's dilemma and the snowdrift game. Ecology Letters, 8:748-766, 2005.

[3] E. Fehr and S. Gächter. Cooperation and punishment in public goods experiments. American Economic Review, 90(4):980-994, 2000.

[4] R. A. Hammond and R. Axelrod. Evolution of contingent altruism when cooperation is expensive. http://www-personal.umich.edu/ axe/. Cited January, 25 th 2006.

[5] T. Kiyonari and P. Barclay. Second-order punishment and reward in public goods games. In 39th Annual Meeting of the Canadian Economics Association, McMaster University, Hamilton, Canada, 2005. Poster presentation.

[6] M. A. Nowak and K. Sigmund. Games on grids. In U. Dieckmann, R. Law, and J. A. J. Metz, editors, The Geometry of Ecological Interactions: Simplifying Spatial Complexity, Cambridge Studies in Adaptive Dynamics, pages 135-150. Cambridge University Press, Cambridge, UK, 2000.

[7] K. Sigmund, C. Hauert, and M. A. Nowak. Reward and punishment. In Proceedings of the National Academy of Sciences of the United States of America, volume 98, pages 10757-10762, 2001. 